\title{
Anterior Commissure Granuloma with Chondronecrosis: A Late Complication of Type I Thyroplasty
}

\author{
Nupur K Nerurkar ${ }^{1}$, Ranjita Krishnan² ${ }^{2}$ Deeksha Agrawal ${ }^{3}$
}

\begin{abstract}
Aim and objective: The development of an anterior commissure granuloma following medialization of the vocal fold with a silicone implant is a rare complication as silicone is a relatively inert material. However, when such granulomas do develop, they are often picked up late due to slow progression over years.

Case description: Our article focuses on the clinical presentation, evaluation, and management of a patient with an anterior commissure granuloma and thyroid cartilage chondronecrosis who presented to us with a history of multiple medialization laryngoplasties in the past, following an immobile vocal fold which was after a parapharyngeal tumor excision.

Conclusion: Though anterior commissure granulomas following medialization laryngoplasty are not frequently seen currently due to the use of implants that produce minimal foreign body reaction and sterile conditions, we believe that in our case the etiology of the granuloma formation with chondronecrosis of the thyroid cartilage may be due to the very large size of the revision implant with resultant extrusion with foreign body reaction leading to long-standing infection.

Clinical significance: Any implant however inert, runs a small risk of infection with granuloma formation which is more likely with large implants and multiple surgeries causing devascularization of tissues. Close observation following thyroplasty with stroboscopy and imaging when needed may help pick up complications early, avoiding progression to chondronecrosis and possibly respiratory distress.

Keywords: Anterior commissure granuloma, Chondronecrosis, Medialization laryngoplasty, Silicone implant, Vocal fold paralysis. International Journal of Phonosurgery \& Laryngology (2020): 10.5005/jp-journals-10023-1186
\end{abstract}

\section{INTRODUCTION}

A granuloma is a submucosal smooth mass that presents as a bulge in the false vocal cord, ventricular mucosa, or true vocal fold and it may also grow inferiorly resulting in a subglottic bulge leading to progressive dysphonia and airway compromise. 'Granulomas are a possible complication of medialization laryngoplasty which uses implants, such as, Teflon, Silicone, Gore-Tex, etc., which evoke an inflammation around the material due to phagocytosis leading to a local giant cell foreign body reaction. ${ }^{2}$ Chondronecrosis of the thyroid cartilage is commonly seen following recurrent perichondritis, long/short-term intubations, infection, trauma, malignancy, or post-radiation. ${ }^{3}$ We discuss a case of anterior commissure granuloma with chondronecrosis of the anterior part of the thyroid cartilage in a patient with a history of multiple medialization laryngoplasties following an immobile vocal fold after a parapharyngeal tumor excision.

\section{Case Description}

A 42-year-old man was referred to our voice center with a history of hoarseness of voice following left vocal fold immobility that had developed as a complication of a left parapharyngeal tumor excision in 2005. He underwent his first medialization laryngoplasty with a silicone implant in September 2006 at an outside center following which his aspiration to liquids improved but his hoarseness persisted. In 2007, a revision medialization laryngoplasty with silicone implant and arytenoid adduction was performed at a different center. Following this, he was asymptomatic for a few years. However, this revised implant was removed in 2011, probably due to a granuloma formation, following which he re-developed hoarseness of voice and was referred to us.

\footnotetext{
${ }^{1-3}$ Bombay Hospital Voice and Swallowing Centre, Bombay Hospital and Medical Research Centre, Mumbai, Maharashtra, India

Corresponding Author: Nupur K Nerurkar, Bombay Hospital Voice and Swallowing Centre, Bombay Hospital and Medical Research Centre, Mumbai, Maharashtra, India, Phone: +91 9821034085, e-mail: nupurkapoor@yahoo.com

How to cite this article: Nerurkar NK, Krishnan R, Agrawal D. Anterior Commissure Granuloma with Chondronecrosis: A Late Complication of Type I Thyroplasty. Int J Phonosurg Laryngol 2020;10(2):54-57.

Source of support: Nil

Conflict of interest: None
}

Stroboscopic examination revealed an anterior commissure granuloma (Fig. 1) with the left vocal fold congested and at a higher level than the right (Fig. 2). Glottic closure was incomplete with a reduced amplitude of the mucosal wave on the left side.

A CT neck with contrast confirmed the laryngoscopic findings and revealed a fat density lesion measuring $8.4 \times 7.7 \mathrm{~mm}$ with peripheral enhancement in the anterior most part of the vocal fold and possible sequestrated thyroid cartilage at the anterior commissure (Fig. 3).

Intraoperatively, the anterior commissure lesion extended more toward the left side and the left vocal fold was confirmed to be at a slightly higher level than the right (Fig. 4). On palpation, the vocal folds were soft but the granuloma was rock hard in consistency. $\mathrm{A} \mathrm{CO}_{2}$ laser excision of the granuloma was performed using an acublade in the super pulse mode following which multiple pieces of necrosed cartilage were noted lying loose as sequestrate anteriorly within frank pus and these sequestrate were carefully 


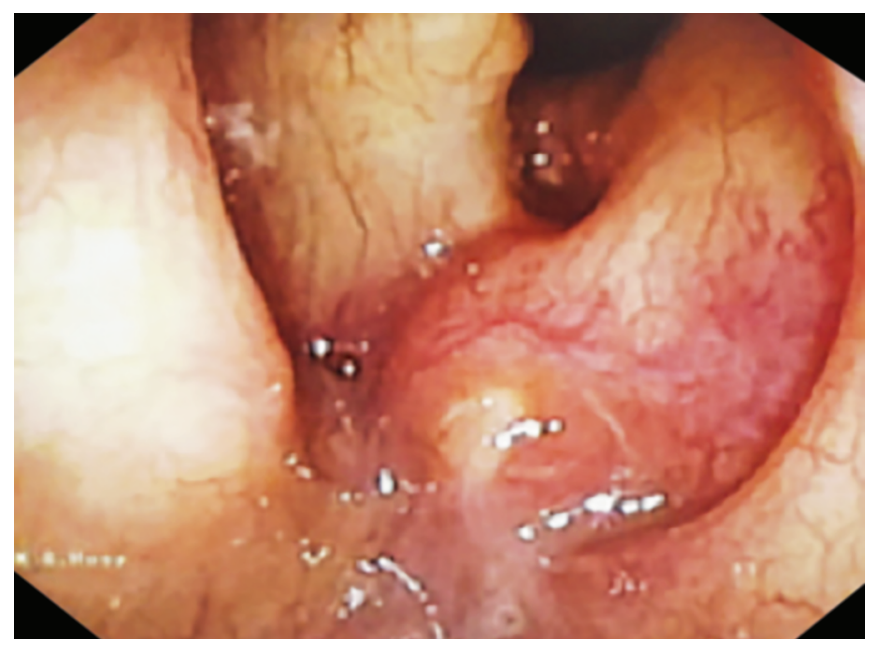

Fig. 1: Preoperative stroboscopic image revealing an anterior commissure granuloma

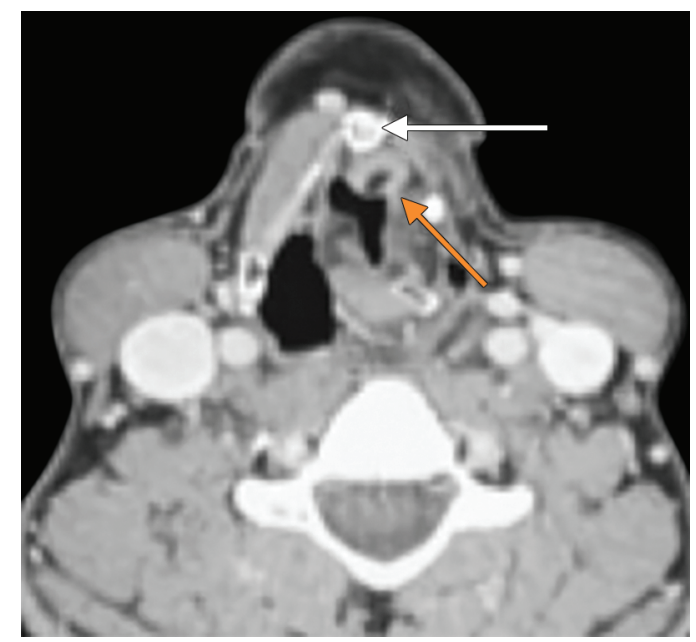

Fig. 3: Computed tomography neck with contrast in axial cross section, revealing the anterior commissure granuloma (red arrow) and possible sequestrated thyroid cartilage fragments (solid white arrow) at the anterior commissure

removed (Fig. 5). A laryngeal wash was given with povidone-iodine and saline to the infected pocket after removal of all necrosed pieces of thyroid cartilage (Fig. 6).

The pus culture and sensitivity revealed Pseudomonas aeroginosa. Histopathology of the granuloma was consistent with fragments of pyogenic inflammatory granulation tissue, dense collagenous tissue, and fibrinous necrotic material.

Our patient was discharged on 3 weeks of oral ciprofloxacin and at 1-month follow-up self-reported significant vocal improvement. Stroboscopic examination revealed an improvement in the amplitude of the left mucosal wave with a symmetrical level of both the vocal folds on adduction (Fig. 7) and good healing at the surgical site (Fig. 8). The patient was then advised voice therapy sessions.

\section{Discussion}

Granulomas following medialization laryngoplasty are not frequently seen currently due to the use of implants that produce minimal foreign body reaction and sterile conditions. The first report of vocal fold medialization dates back to 1911, by Brunings,

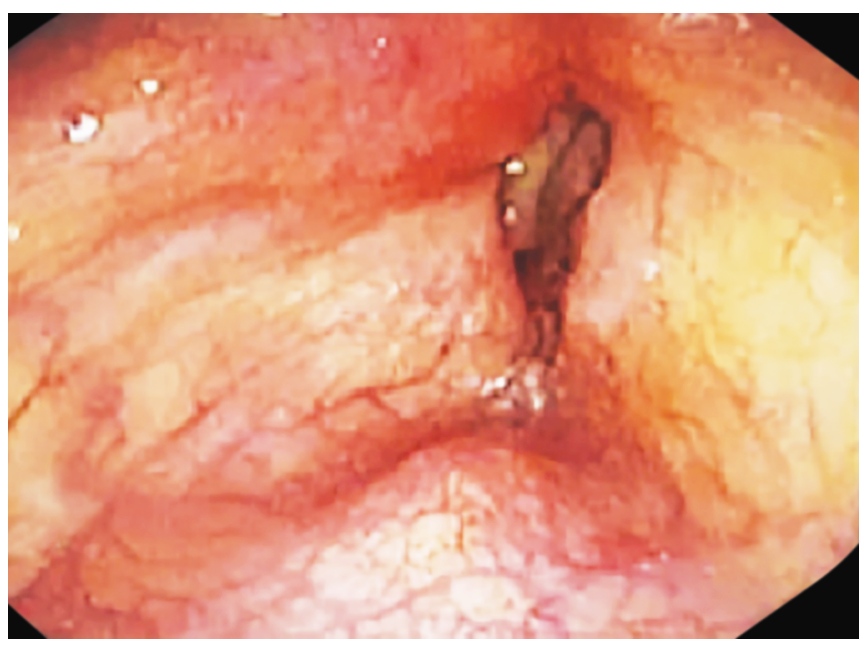

Fig. 2: Preoperative stroboscopic image during phonation, revealing a phonatory gap with the left vocal fold at a higher level than the right

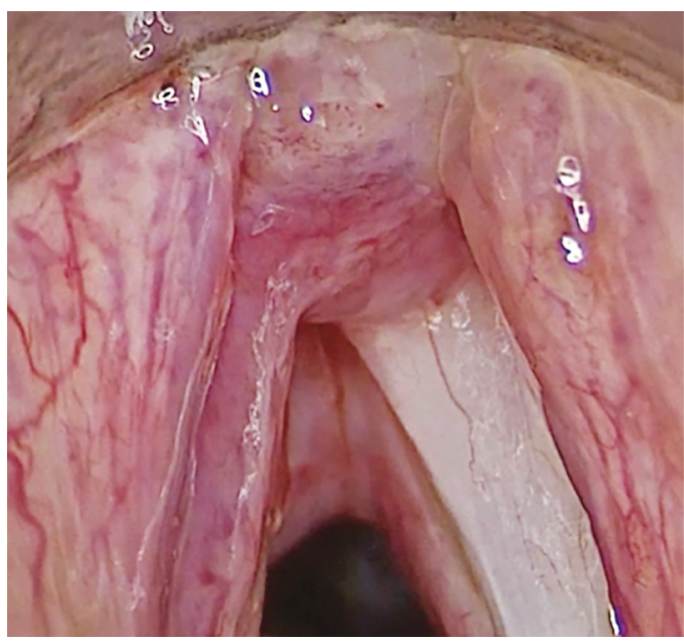

Fig. 4: Intraoperative image demonstrating the anterior commissure lesion extending more toward the left side and left congested vocal fold, at a slightly higher level than the right vocal fold

who used paraffin to successfully treat patients with vocal fold paralysis. ${ }^{2,4}$ Of the materials used for medialization laryngoplasty, Teflon was considered an ideal material initially, as it was thought to be biologically stable, but it immediately evokes inflammation that leads to a local giant-cell foreign-body reaction. ${ }^{2,5}$ Silicone, on the contrary, is an inert material resulting in less scarring of the cordal mucosa and disruption of mucosal wave in comparison to Teflon. Silicone implants may, however, migrate to the endolarynx causing potentially dangerous situations, such as, aspiration of the implant or externally out of the thyroid cartilage, losing the medialization effect of the procedure. ${ }^{6}$ Autologous fat is the only material proved not to cause local inflammation, but is highly unstable through time due to physiological reabsorption. ${ }^{2}$

Chondronecrosis of the thyroid cartilage is a grave complication, which is commonly seen following recurrent perichondritis of the thyroid cartilage, long/short-term intubations, infection, trauma, malignancy, or post-radiation. Hence, early detection is necessary to prevent its progression from perichondritis to chondritis and necrosis. ${ }^{3}$

We believe that in our case the etiology of the granuloma formation with chondronecrosis of the thyroid cartilage may be 


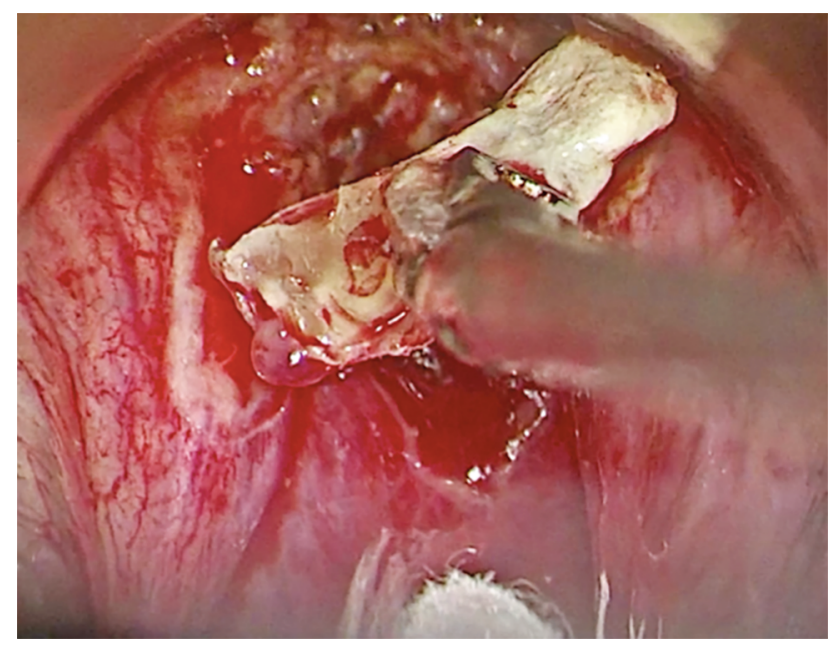

Fig. 5: Intraoperative image showing a piece of necrosed thyroid cartilage that was lying loose as sequestra and is being removed with a crocodile forceps

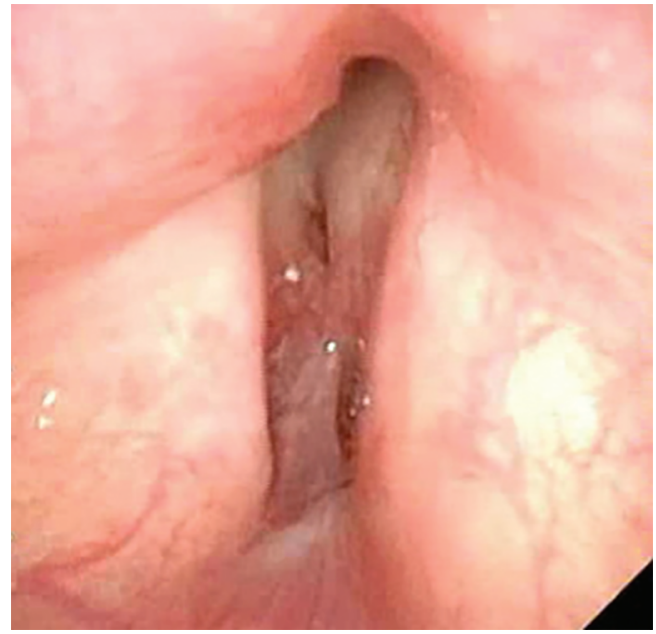

Fig. 7: Stroboscopic image at one-month follow-up showing symmetrical level of both the vocal folds on adduction

due to the very large size of the revision implant with resultant extrusion with foreign body reaction leading to long-standing infection.

Voice in patients who develop granulomatous lesions is characterized by an abnormally low pitch, forced loudness, and a strained hoarse quality ${ }^{7}$ and videostroboscopy are quite consistent revealing a stiff, non-vibratory vocal fold mass as was seen in our patient.

A CT scan of the neck with contrast plays an important role in distinguishing between various types of vocal cord augmentations and their complications can be picked up by combined use of positron emission tomography-computed tomography (PET-CT). ${ }^{8}$ It also helps in assessing the exact location and extent of granuloma formation.

During transoral endoscopic laser excision of the granuloma, the necrosed pieces of cartilage were lying loose as sequestrate within frank pus and its removal resulted in quick healing with a resolution of vocal fatigue and vocal improvement.

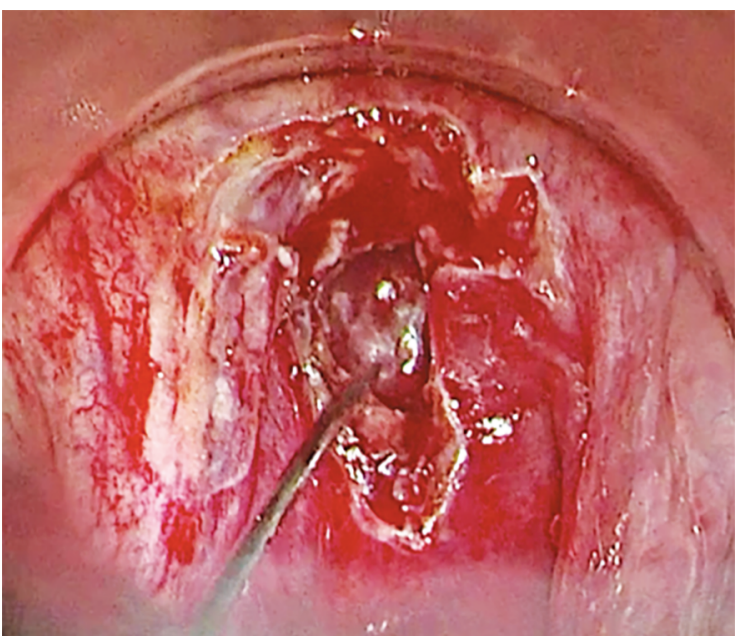

Fig. 6: A laryngeal wash being given with povidone iodine and saline to the infected pocket after removal of all necrosed pieces of thyroid cartilage

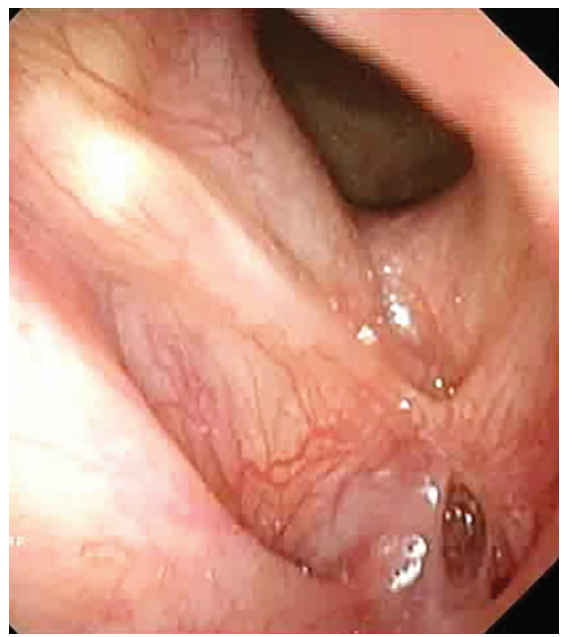

Fig. 8: Stroboscopic image at one-month follow-up showing good healing at the surgical site

The management of granulomas may differ with Billante et al. preferring excision of a Teflon granuloma by lateral laryngotomy, ${ }^{7}$ Russell et al. proposed cordectomy as an alternative surgical approach for managing Teflon granuloma, ${ }^{9}$ and Varvares et al. adopted a technique of laser incision into the superior aspect of the Teflon implant, followed by vaporization and preservation of a margin of the mucosa of the cord medially. ${ }^{10}$

\section{Conclusion}

Our case demonstrates a late complication of a silicone implant where a long-standing infection resulted in thyroid cartilage chondronecrosis. A correct diagnosis is essential to manage these tricky cases. We could successfully remove all the infected segments of thyroid cartilage sequestrate along with the granuloma, which helped in resolving the localized inflammation, bringing the vocal folds to a symmetrical position with subsequent voice improvement. 


\section{Clinical Significance}

Any implant, however inert, runs a small risk of infection with granuloma formation which is more likely with large implants and multiple surgeries causing de-vascularization of tissues. Close observation of the patient following thyroplasty with stroboscopy and imaging when needed may help pick up complications early, avoiding progression to chondronecrosis and possibly respiratory distress.

\section{References}

1. Simpson B, Rosen C. Endoscopic management of teflon granuloma. Operat Techniq Laryngol 2008. 151-154.

2. Marco Bottazzoli MD, Alessandra Marcantoni MD, Lorena Picori MD, et al. Metachronous malingnancy, 27 years after teflon cordal injection. Arch Clin Med Case Rep 2019;3(6):442-450.

3. Halkud R, Shenoy AM, Naik SM, et al. Chondroradionecrosis of larynx a delayed complication of radiotherapy: management and review of literature. Indian J Surg Oncol 2014;5(2):128-133. DOI: 10.1007/ s13193-014-0315-6.
4. Bruning W. Uber eine neue behandlungsmethode der rekurrenslahmung. Verhandlungen des Vereins Deuischer Laryngologen 1911;18:93-151.

5. Yeretsian RA, Blodgett TM, Branstetter IV BF, et al. Teflon-induced granuloma: a false-positive finding with $\mathrm{PET}$ resolved with combined PET and CT. AJNR Am J Neuroradiol 2003;24(6):1164-1166.

6. Van Ardenne N, Vanderwegen J, Van Nuffelen G, et al. Medialization thyroplasty: vocal outcome of silicone and titanium implant. Eur Arch Otorhinolaryngol 2011;268(1):101-107. DOI: 10.1007/s00405-0101327-7.

7. Billante CR, Burkard KA, Clary JM, et al. Voice outcome following lateral laryngotomy to remove teflon granuloma. ORL J Otorhinolaryngol Relat Spec 2001;63(5):302. DOI: 10.1159/000055763.

8. Kumar VA, Lewin JS, Ginsberg LE. CT assessment of vocal cord medialization. AJNR Am J Neuroradiol 2006;27(8):1643-1646.

9. Russell JD, Perry A, Cheesman AD. Cordectomy: a solution to teflon granuloma of the vocal fold. J Laryngol Otol 1995;109(1):53-55. DOI: 10.1017/S0022215100129226.

10. Varvares MA, Montgomery WW, Hillman RE. Teflon granuloma of the larynx: etiology, pathophysiology, and management. Ann Otol, Rhino Laryngol 1995;104(7):511-515. DOI: 10.1177/000348949510400702. 